\title{
Familial Mediterranean fever: prevalence, penetrance and genetic drift
}

\author{
Ruth Gershoni-Baruch*,1,3 ${ }^{*}$ Marwan Shinawi ${ }^{1}$, Kasinetz Leah ${ }^{1}$, Khader Badarnah ${ }^{1}$ and \\ Riva Brik $^{2,3}$ \\ ${ }^{1}$ Institute of Human Genetics, Rambam Medical Center, Haifa, Isreal; ${ }^{2}$ Department of Pediatric Rheumatology, \\ Rambam Medical Center, Haifa, Israel; ${ }^{3}$ Bruce Rappoport Faculty of Medicine, Technion-Israel Institute of \\ Technology, Haifa, Israel
}

FMF is widely distributed in populations inhabiting the Mediterranean basin. It is mainly attributed to five founder mutations (M680I, M694V, M694I, V726A, E148Q) in the MEFV gene. The frequencies and distribution of these mutations in 146 FMF patients, of Arab and Jewish descent, were compared to that observed in 1173 healthy individuals of pertinent ethnic groups. Five mutations accounted for $91 \%$ of FMF chromosomes in our patients. Mutation M694V, predominant in North African Jews, was observed in all patients other than Ashkenazi Jews; mutation V726A was prevalent among all patients other than North African Jews; mutations M694I and M680I were mainly confined to Arab patients. Overall carrier rates, for four mutations (M680I, M694V, V726A, E148Q), were extremely high in our healthy cohort composed of Ashkenazi ( $n=407)$; Moroccan ( $n=243)$; Iraqi Jews $(n=205)$; and Muslim Arabs $(n=318)$; calculated at $1: 4.5$; $1: 4.7 ; 1: 3.5$ and $1: 4.3$ respectively. The V726A allele prevalent among Ashkenazi and Iraqi Jews and Muslim Arabs (carrier rates: 7.4, 12.8 and 7.3\%, respectively) was not found among Moroccan Jews. The M694V allele detected among Moroccan and Iraqi Jews and Muslim Arabs (carrier rates 11.1, 2.9 and 0.6\%, respectively) was not observed among Ashkenazim. The overall frequency of mutations V726A and E148Q in Ashkenazim, Iraqi Jews and Arabs indicates that the bulk of individuals that comply with the genetic definition of FMF remain asymptomatic. European Journal of Human Genetics (2001) 9, 634-637

Keywords: FMF; MEFV; genetic drift; population; carrier rate

\section{Introduction}

Familial Mediterranean fever (FMF) is an autosomal recessive disorder (MIM\# 249100) characterised by multiple attacks of serosal inflammation. It affects primarily people of Mediterranean extraction, mostly Sephadic Jews, Armenians, Arabs and Turks. ${ }^{1,2}$ It is very common in the populations at risk with estimated carrier rates of $1 / 6$ in Armenians, $1 / 7$ in North African Jews and 1/13 in Iraqi Jews. ${ }^{3,4}$

The gene causing FMF (MEFV) has been cloned and five common founder mutations (M694V, V726A, M680I, M694I,

\footnotetext{
*Correspondence: R Gershoni-Baruch, Institute of Human Genetics, Rambam Medical Center, Haifa, Israel.

E-mail: rgershoni@rambam.health.gov.il

Received 11 January 2001; revised 2 April 2001; accepted 1 May 2001
}

E148Q), each segregating with one ancestral haplotype, were identified. $^{5-8}$ SNP haplotype data, recently presented, is consistent with the opinion that each of these mutations share a common progenitor. ${ }^{8}$

To better define the distribution and penetrance of MEFV mutations in our heterogeneous population consisting of Jews and Arabs we tested 146 unrelated FMF patients and compared the frequencies and distribution of these mutations in our patients to that observed in 1173 individuals, of pertinent ethnic groups, unselected for personal or family history of FMF.

\section{Subjects and methods}

Blood samples were obtained from 146 unrelated patients strictly complying with FMF clinical diagnostic criteria, ${ }^{9}$ who 
attended our Rheumatology clinic between 1998 and 2000, and 1173 healthy individuals who presented for heterozygote detection of recessive diseases (cystic fibrosis and other). Patients' group included 75 Jewish (50 North African, three Ashkenazi, 12 non-Ashkenazi, 10 of mixed Jewish descent) and 71 Arab (56 Muslim, 10 Christian, five Druze) individuals. The healthy population included individuals who previously signed a consent form allowing the use of their DNA for research purposes and for whom DNA was available. It was composed of Ashkenazi $(n=407)$, Iraqi $(n=205)$ and Moroccan Jews (243) and 318 Muslim Arabs. The study had the approval of the Ethical Committee at Rambam Medical Center. Patients' blood samples were systematically screened for the five founder mutations (M680I, M694V, M694I, V726A, E148Q). When patients of mixed Jewish origin were found to carry two different mutations, we analysed one of the two parents in order to determine the exact origin of the mutations. The healthy control population was differentially screened for the four most common mutations (M694V, M680I, V726A and E148Q). We did not screen the healthy population for mutation M694I, which is rather rare and preferentially distributed among our patients of Druze and Christian Arab origin (data not shown).

\section{Mutation analysis}

Mutations V726A and E148Q create a constitutive restriction site. They were distinguished by PCR amplification followed by digestion with appropriate enzymes made to distinguish the wild type allele from the mutant allele, as previously described. 8,10

Mutation M680I (either a or b) abolishes a constitutive restriction site. PCR amplification followed by digestion with Hinf-I distinguishes the wild type allele from the mutant allele. ${ }^{8,10}$

Mutations M694V and M694I were detected by PCR amplification with specific primers that produce a modified restriction enzyme digest made to distinguish the wild type allele from the mutant allele, as previously described. ${ }^{10}$

\section{Statistical analysis}

The proportion of individuals positive for the mutated alleles was calculated for each ethnic subgroup (Ashkenazi, Iraqi and Moroccan Jews and Moslem Arabs) and a 95\% CI, based on the binomial distribution, was determined. In nine individuals who carried more than one mutation, each mutation was counted separately.

\section{Results}

Distribution of founder mutations in FMF patients

Ninety-one per cent of mutation bearing chromosomes from 146 unrelated Jewish $(n=75)$ and Arab $(n=71)$ patients were characterised (Table 1). Mutation M694V identified 94\% of FMF chromosomes from patients of North African Jewish descent; 33\% of Iraqi Jewish FMF chromosomes and $14.8 \%$ of Arab FMF chromosomes. It was not observed among Ashkenazi Jews. Mutation V726A accounted for 33\% of Iraqi Jewish FMF chromosomes, 29.6\% of Arab FMF chromosomes and was not found in North African Jews. Mutations M680I and M694I were observed in Arab patients only and accounted for 18.3 and $8.4 \%$ of Arab FMF chromosomes, respectively (Table 1). All in all, three mutations (M694V, V726A and M680I) accounted for 63\% (89/142) of Arab FMF chromosomes and two of them (M694V and V726A) identified 89\% (133/150) of Jewish FMF chromosomes.

Frequency and distribution of founder mutations in the general population (Table 2)

One hundred and ninety-one mutated alleles were identified in 181 individuals, nine of whom had two mutated alleles (three compound M694V/V726A heterozygotes; two V726A homozygotes; one M680I homozygote; one compound M680I/V726A heterozygote; one compound E148Q/V726A heterozygote; one compound E148Q/M694V heterozygote).

FMF carrier frequencies were extremely high in all populations studied. The frequency of mutation M694V was highest in Moroccan Jews - 0.55 (95\% CI=0.37-0.8). It was prevalent among Iraqi Jews- 0.15 (95\% CI=0.05-0.32), rare among Muslim Arabs-0.03 (95\% CI=0.004-0.11) and absent from 814 Ashkenazi chromosomes tested. The frequency of mutation V726A was high in Iraqi Jews-0.75 (95\% CI=0.50-0.11), Ashkenazi Jews-0.37 (95\% CI=0.25$0.53)$ and Muslim Arabs-0.38 (95\% CI=0.25-0.56); It was not found among Moroccan Jews. The frequency of mutation M680I in Muslim Arabs was-0.13 (95\% CI=0.05-0.25). It was not observed in the Jewish population. Mutation E148Q was ubiquitous and common in all ethnic groups studied, with frequencies of $-0.55(95 \% \mathrm{CI}=0.28-0.96)$ in Moroccan Jews; $-0.7(95 \% \mathrm{CI}=0.39-1.15)$ in Iraqi Jews; $-0.75(95 \%$ $\mathrm{CI}=0.5-1.07)$ in Ashkenazi Jews and-0.65 (95\% CI=0.35 1.1) in Muslim Arabs.

\section{Discussion}

FMF follows an autosomal recessive pattern of inheritance, yet a significant proportion of patients have no known affected relatives. Estimated carrier rates vary from 1/6-1/7 in North African Jews, $1 / 13$ in Iraqi Jews to $1 / 135$ in Ashkenazi Jews. ${ }^{3,4,11}$

It has been repeatedly shown, that the variable disease phenotype, severe in North African Jews, milder in Iraqi Jews and Druzes is at least partly due to allelic heterogeneity with mutation M694V being associated with a severe phenotype and amyloidosis, and mutation V726A with a milder form of the disease. $^{12-14}$

The five common founder mutations are differentially distributed among our patients: M694V, predominant among North African Jews, was detected in all patients other than Ashkenazi Jews; V726A was observed in all patients other 
Table 1 Frequency of founder mutations in FMF patients

No* M694V V726A M694I M680I E148Q Null

\begin{tabular}{lrrrrrrr} 
North African Jews & 118 & 111 & 0 & 0 & 0 & 5 & 2 \\
Iraqi Jews & 15 & 5 & 5 & 0 & 0 & 3 & 2 \\
$\begin{array}{l}\text { Non-Ashkenazi Jews } \\
\text { Ashkenazi Jews }\end{array}$ & 10 & 5 & 2 & 0 & 0 & 2 & 1 \\
Total & $\mathbf{1 5 0}$ & 0 & 5 & 0 & 0 & 1 & 1 \\
Moslem Arabs & 112 & 18 & 35 & 6 & 25 & 14 & 14 \\
Christian Arabs & 20 & 1 & 4 & 3 & 1 & 0 & 1 \\
Druze Arabs & 10 & 2 & 3 & 3 & 0 & 6 & 6 \\
Total & $\mathbf{1 4 2}$ & $\mathbf{2 1}$ & $\mathbf{4 2}$ & $\mathbf{1 2}$ & $\mathbf{2 6}$ & $\mathbf{2 0}$ & $\mathbf{2 1}$ \\
\hline
\end{tabular}

*Number of chromosomes tested.

Table 2 Carrier rates of FMF founder mutations in the general Israeli population

\begin{tabular}{llllll}
\hline Mutation & Ethnic group & Tested $^{a}$ & Positive & Per cent & $95 \% \mathrm{Cl}$ \\
\hline \multirow{2}{*}{ M694V } & Moroccan Jewish & 486 & 27 & 5.56 & $3.69-7.98$ \\
& Iraqi Jewish & 410 & 6 & 1.46 & $0.54-3.16$ \\
& Ashkenazi Jewish & 814 & 0 & 0.0 & $0.00-0.45$ \\
& Muslim Arab & 636 & 2 & 0.31 & $0.04-1.13$ \\
V726A & Moroccan Jewish & 362 & 0 & 0.00 & $0.00-1.01$ \\
& Iraqi Jewish & 374 & 28 & 7.49 & $5.03-10.64$ \\
& Ashkenazi Jewish & 808 & 30 & 3.71 & $2.52-5.26$ \\
& Muslim Arab & 632 & 24 & 3.80 & $2.45-5.60$ \\
\multirow{4}{*}{ M680I } & Moroccan Jewish & 286 & 0 & 0.00 & $0.00-1.28$ \\
& Iraqi Jewish & 132 & 0 & 0.00 & $0.00-2.76$ \\
& Ashkenazi Jewish & 158 & 0 & 0.00 & $0.00-2.31$ \\
& Muslim Arab & 626 & 8 & 1.28 & $0.55-2.50$ \\
& & & & & \\
E148Q & Moroccan Jewish & 200 & 11 & 5.50 & $2.78-9.63$ \\
& Iraqi Jewish & 200 & 14 & 7.00 & $3.88-11.47$ \\
& Ashkenazi Jewish & 362 & 27 & 7.46 & $4.97-10.67$ \\
& Muslim Arab & 198 & 13 & 6.54 & $3.54-10.97$ \\
\hline
\end{tabular}

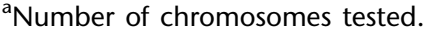

than North African Jews; M680I is common and confined to Arab patients; M694I is rare and confined to Arab patients; E148Q is occasionally encountered in patients from all ethnic groups.

Our population based study shows that the overall carrier frequencies, of the mutations tested, are extremely high in all ethnic groups studied. Three mutations taken together (M694V, M680I, V726A) were observed at rates that vary from 1:6 in Iraqi Jews, 1:9 in Moroccan Jews, $1: 13$ in Ashkenazi Jews and 1:10 in Arabs. The carrier rate of mutation E148Q ranged from 10 to $13 \%$ in all populations studied, thereby exceeding that calculated for all other mutations taken together. In patients, however, the E148Q mutation, accounted for only $8.9 \%$ of mutated alleles, in line with the opinion that considers this mutation to be of reduced penetrance. ${ }^{7}$ Overall carrier rates for the four most common FMF alleles reached $1: 3.5$ in Iraqi Jews, $1: 4.7$ in Moroccan Jews, $1: 4.5$ in Ashkenazi Jews and $1: 4.3$ in Arabs. These rates do not take into account other, rare, mutations recently described..$^{7-9}$

The prevalence of mutation V726A, among Ashkenzim, by far exceeds that calculated by disease prevalence, indicating that the bulk of Ashkenazi individuals who comply with the genetic diagnosis of FMF remain asymptomatic or undiagnosed. Among our patients of non-Ashkenazi or Arab origin the V726A allele was detected at rates five times lower than expected based on our calculated carrier frequencies. The high disease prevalence of FMF in non-Ashkenazi Jews should be mainly attributed to mutation $\mathrm{M} 694 \mathrm{~V}$ known to be associated with a severe phenotype. Although, differences in lifetime cumulative penetrance are by now attributed to different mutations, the V727A mutation remains practically totally silent and non-penetrant in Ashkenazi Jews. Whether this is due to modifier genes or environmental factors remains to be established.

\section{References}

1 Sohar E, Gafni J, Pras M, Heller J: Familial Mediterranean fever: a survey of 470 cases and review of the literature. Am J Med 1967; 43: $227-253$.

2 Pras M, Bronshpigel N, Zemer D, Gafni J: Variable incidence of amyloidosis in familial Mediterranean fever among different ethnic groups. Johns Hopkins Med J 1982; 150: 22-26.

3 Daniels M, Shohat T, Brenner-Ullman A, Shohat M: Familial Mediterranean fever: High gene frequency among the nonAshkenazi and Ashkenazi-Jewish populations in Israel. Am J Med Genet 1995; 55: $311-314$.

4 Rogers DB, Shohat M, Petersen GM et al: Familial Mediterranean fever in Armenians. Autosomal recessive inheritance with high gene frequency. Am J Med Genet 1989; 34: 168-172.

5 French FMF Consortium: A candidate gene for familial Mediterranean fever. Nature Genet 1997; 17: 25 - 31.

6 International FMF Consortium: Ancient missense mutations in a new member of the RoRet gene family are likely to cause familial Mediterranean fever. Cell 1997; 17: 25-31.

7 Bernot A, da Saliva C, Petit J-L et al: Non-founder mutations in the MEFV gene establish this gene as the cause of familial Mediterranean fever (FMF). Hum Mol Genet 1998; 7: 1317-1325.

8 Aksentijevich I, Torosyan Y, Samuels J et al: Mutation and haplotype studies of familial Mediterranean fever reveal new ancestral relationships and evidence for a high carrier frequency in the Ashkenazi Jewish population. Am J Hum Genet 1999; 64: 949-962.

9 Livneh A, Langevitz P, Zemer D et al: Criteria for the diagnosis of familial mediterranean fever. Arthritis Rheum 1997; 40: 18791885.

10 Gershoni-Baruch R, Kepten I, Shinawi M, Brik R: Direct detection of common mutations in familial mediterranean fever gene (MEFV) using naturally occurring and primer mediated restriction fragment analysis. Hum Mutation (in brief \#) 257; 1999 .

11 Yuval Y, Hemo-Zisser M, Zemer D, Sohar E, Pras M: Domonant inheritance in two families with familial Mediterranean fever (FMF). Am J Med Genet 1995; 57: 455 - 457. 
12 Dewalle M, Domingo C, Rozenbaum M et al: Phenotypegenotype correlation in Jewish patients suffering from familial Mediterranean fever (FMF). Eur J Hum Genet 1998; 6: 95 - 97.

13 Shohat M, Magal N, Shohat $\mathrm{T}$ et al: Phenotype-genotype correlation in familial Mediterranean fever: evidence for an association between Met694Val and amyloidosis. Eur J Hum Genet 1999; 7: 287-292.
14 Cazeneuve C, Sarkisian T, Pecheux C et al: MEFV-gene analysis in Armenian patients with familial Mediterranean fever: diagnostic value and unfavourable renal prognosis of the M694V homozygous genotype-Genetic and therapeutic implications. Am J Hum Genet 1999; 65: 88-97. 